\title{
Qualitative exploration of dental and health care personnel's awareness of signs displayed in victims of torture with focus on the oral cavity
}

\author{
Karolina Willix ${ }^{1,2}$, Evelina Ekman ${ }^{1,2}$, Karin Klefbom³ ${ }^{3}$ Lena Karlsson²
}

\section{Key points of interest}

- Numerous torture victims have experienced severe physical and psychological trauma to their face, mouth, and teeth; areas that dental visits involve. Survivors of torture carry a risk of reliving the trauma during a visit to the dentist, if the dental personnel do not know the background of individual and are not aware of signs of torture with focus on the oral cavity.

- The importance of educated health care personnel at resource centers for asylum seekers or refugees, and dental personnel who can provide oral health services to torture survivors and minimizing the risk of re-traumatization is highlighted in this study, as well as the importance of multidisciplinary collaboration.
1) First authors

2) Department of Dental Medicine, section of Cariology, Karolinska Institutet, Sweden

3) Transcultural Center, region Stockholm, Sweden Correspondence to:

Lena Karlsson lena.karlsson@ki.se

\begin{abstract}
Introduction: Numerous torture victims have experienced severe physical or psychological trauma to their face, mouth, and teeth. A dental visit carries a risk for torture survivors to relive the trauma, since the situation may trigger a recollection of previous suffering. Although health care personnel at resource centers for torture victims are equipped with various tools to help and assist these individuals in their rehabilitation, very few centers have protocols in place to refer out victims to dental professionals with experience and knowledge in the area. The aim of this study was to investigate the extent of dental and health care personnel's knowledge and awareness to detect various torture signs, with focus on the oral cavity.

Material and Methods: Participants included 16 dental and 6 health care personnel. Qualitative data was collected from participants from individual and group interviews and responses to a questionnaire. All interviews were transcribed, and a phenomenological-hermeneutical method was used to analyse the participants' answers.

Results: Dental personnel demonstrated a lack of knowledge and experiences regarding signs of torture with focus on the oral cavity and indicated lack of experiences with multidisciplinary collaboration in the care of torture victims. Most of the health care personnel had
\end{abstract}


clear suggestions for external signs of torture and showed good knowledge of how to refer a torture victim to dental personnel.

Discussion: Through a multidisciplinary approach, better health care resources utilization can be achieved and potential harmful longterm effects resulting from dental care visits can be prevented. There is a need for increased awareness and knowledge among dental and health care personnel of injuries and signs resulting from torture in the oral cavity.

Keywords: torture, oral cavity, dental personnel, health care personnel

\section{Introduction}

Numerous torture victims have experienced severe physical and psychological trauma to their face, mouth, and teeth; areas that dental visits involve (Abu-Awwad et alt (2020), Caldas et alt (2010)). Survivors of torture carry a risk of reliving the trauma during a visit to the dentist, a situation that may trigger recollection of previous suffering (Hoyvik et alt (2019), Singh et alt (2008), Keller et alt (2014)).

Hoyvik et alt (2019) studied torture experiences, symptoms of post-traumatic stress disorder (PTSD), and dental anxiety among refugees. The study found that $62 \%$ of the torture survivors have experienced torture related to their face, $35 \%$ related to their mouth and $23 \%$ related to their teeth. The odds of being highly dentally anxious was 6.1 times higher in refugees with torture experience and 9.3 times higher in torture victims with PTSD symptoms, compared to refugees with no history of torture. Additionally, among the female refugees, $31 \%$ had been sexually assaulted, which is a known risk factor for dental anxiety when the abuse involved oral penetration.

The Istanbul protocol (UN Office of the High Commissioner for Human Rights
(2004)) reports that known torture methods against the oral cavity includes, for instance, extraction and grinding/breaking of teeth, or application of electric current to teeth. The application of electric current can lead to several negative conditions, including loss or rupture of the teeth, mandibular fractures, subluxation of the mandibula due to the muscle spasms arising from electric currents, dental filling fractures, displaced fillings, and tooth ache (UN Office of the High Commissioner for Human Rights (2004), Di Napoli et alt (2005), Ozkalipci (2010)). Torture may also include sexual abuse to the oral cavity which can trigger lesions in the oral cavity by having objects or materials forced into the mouth (Hoyvik, A., Lie, B., \& Willumsen, T. (2019)). Lesions and scars can likewise be detected if the victim has been subjected to chemical torture or/by being forced to swallow toxins, such as the chemical thallium or drugs (UN Office of the High Commissioner for Human Rights (2004), Di Napoli et alt (2005)). Waterboarding, another form of torture, where the torturer pours water onto the victim's face and their breathing passages, triggers an immediate gag reflex and a drowning sensation for the victim. This can produce extremely strong near-death feelings and is considered one of the most severe experiences a human being may encounter (Ozkalipci et alt (2010)). Sára et alt (2014) conducted clinical forensic examinations of alleged torture victims $(n=33)$ at the University of Copenhagen and direct impact to the teeth was reported in four cases. In three of four cases, it was reported that teeth had been extracted with pliers and in the fourth case, teeth had been grinded down with the force of a file. These methods are also consistent with torture methods that Ozakaplici et al (2010) reported in the The Atlas of Torture.

The oral cavity is a highly private area of the body and regular dental visits often involve sharp dental instruments, water in the patient's 
mouth, and actions that can trigger gag-reflexes; sensations that are often harmless for the regular patient, but can be triggers for a torture victim, forcing them to relive painful and distressing memories. Any stressful and anxious situation caries the risk of harming the rehabilitation process of a torture victim, and although health care personnel at resource centers are equipped with various tools to help and assist torture victims in their rehabilitation, very few centers have protocols in place to refer out victims to dental personnel with experience and knowledge about trauma triggers. As a result, most torture victims end up in general dental care, something that can potentially be harmful to the victim's rehabilitation process. Singh H et alt (2008) confirmed in their study the importance of educated dental personnel who can provide oral health services to torture survivors without the risk of re-traumatization.

Increased knowledge and awareness will hopefully encourage investigations and discussions with patients to figure out the best way to proceed with dental treatments, based on the victim's rehabilitation process, injuries, traumas, and other information that has been presented. Through a multidisciplinary approach, potential harmful, long-term effects resulting from dental care visits can be prevented and the approach can additionally contribute to better resource utilization in health care.

The aim of this study was to investigate the extent of dental and health care personnel's knowledge and awareness of how to detect various torture signs with a focus on the oral cavity.

\section{Material and Method}

\section{Study group}

A purposive sampling was carried out, and in total, 39 health care personnel with specialised knowledge in the subject of torture and 5 clinics with a total of 16 clinical working licensed dental staff were invited to take part in the study. The health care personnel consisted of doctors or psychologists working at the Transcultural Center, a resource center for asylum seekers or refugees in Stockholm, Sweden. The health personnel in this study worked at a specialist service for asylum seekers and has a commitment to provide support to health and dental care personnel in

Figure 1. Professions and employment sectors of the participants

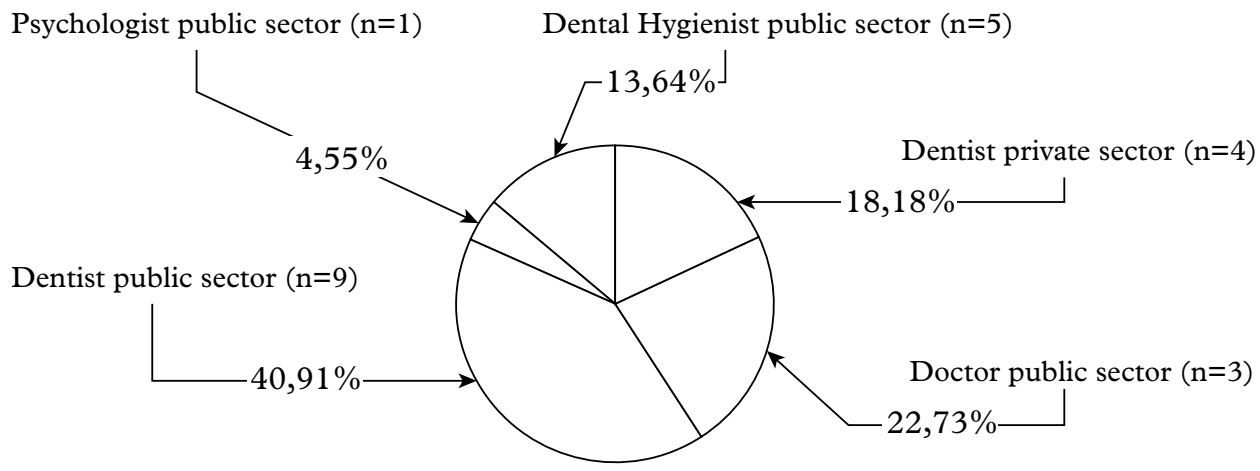


issues of culture, migration and refugee status. The licensed dental staff group consisted of dentists or dental hygienists working at generalist practices in the private or public sector within the municipals of Stockholm, Sweden. The participants were recruited via our own or our colleagues' networks, as well as through the internet. All invitees received verbal and written information from the researchers. The inclusion criteria were: i) health care personnel with specialised knowledge of the subject torture, and ii) licensed dental personnel. The exclusion criteria were: i) not licensed medical and dental staff, and ii) working outside the city of Stockholm. Figure 1 for a breakdown of the professions and employment sectors of the participants.

\section{Ethical considerations}

Sixteen dental and 6 health care personnel signed informed consent forms and accepted to participate in the study and were consecutively enrolled after informed consent. The study was approved by Karolinska Institutet, Sweden and was conducted according to the World Medical Association Declaration of Helsinki, Ethical Principles for Medical Research Involving Human Subjects (World Medical Association (2021)). All participants signed an informed consent.

\section{Study design}

This study was based on individual interviews, group interviews, and quantitative data from a questionnaire. A questionnaire with 6 closed questions were handed out to all participants ( $n=22$ ) before the interviews.

The survey questions were the same for both dental and health care personnel, with the exception being that the answer choices for question 2 differed. Table 1a and Table 1b

The interviews were conducted by $\mathrm{KW}$ and EE during the year 2020, and the inter- view location varied, either at the participants' clinics or in their offices. Each interview lasted approximately half an hour and was audio recorded and transcribed verbatim. The interviews were semi-structured and thematic. An interview guide was used, which consisted of six main questions and subsequent follow-up questions, with the same interview questions for all participants.

The aim was for the main questions to lead to a discussion. If that was not the case, sub-questions were used. The main questions asked to the dental personnel were about their experiences of finding atypical damage to the oral cavity, how often they saw such atypical injuries, how they approached such a situation, what they know about the oral cavity in the context of torture, and whether they think that general dentists have knowledge to identify, treat and work with a torture victim. The main questions asked to the health care personnel were about how the torture victim gets in contact with them, how common it is that the patient has atypical damage to the oral cavity, what experience they have about the importance of the oral cavity in a torture situation, how they approach such a situation (referral to a dentist etc.), and what is their experience regarding treatment and rehabilitation of torture injuries in the oral cavity. Follow-up questions were asked when needed. For example, the question of "How often do you see atypical injuries to patients in the oral cavity?", was followed up with "Do you investigate the cause or background of the injury? If the answer was, yes, they were asked, "How do you do this?" If the answer was no, they were asked "Why not?"

The audio recordings, quotes and notes taken were translated from Swedish to English.

Analysis

The qualitative content analysis was made ac- 
Table 1a. Survey Questions, dental personnel

Karolinska Institute

\section{Survey Questions, dental professionals:}

1) Do you meet asylum seeking patients or refugee patients on a daily basis?

YES, how many patients per day

NO, estimate how often

2) In your opinion, what does asylum seeking patients or refugee patients primarily seek help for?

$\square$ Immediate pain

$\square$ Restorative Treatment

Prosthetics

$\square$ Other:

3) How aware are you about the effects of torture for the victim?

$\square$ Very aware $\square$ Aware
Somewhat aware
Not aware at all

4) How common do you think that torture against the oral cavity/face is?

$\begin{array}{ll}\square \text { Very common } & \square \text { Common } \\ \square \text { Not very common } & \square \text { Not common }\end{array}$

5) Is torture something that you have gained knowledge about through your education or at your workplace?

$\square$ Yes, I have received information about torture at my workplace

$\square$ Yes, I have received information about torture through my education

$\square$ We have discussed the subject to some extent (at the workplace or through education)

$\square$ No, I have not received any information about the subject at my workplace

$\square$ No, I have not received any information about the subject through my education

6) Is being a torture victim a reason to seek asylum?

$\square$ Yes $\square$ No

Information about you:

- Age:

- Number of years as a professional:

- Profession:

Working today in:

Public Sector

Private Sector 
Table 1b. Survey Questions health care personnel

Karolinska Institutet

\section{Survey Questions health care personnel:}

1) Do you meet asylum seeking patients or refugee patients on a daily basis?

YES, how many patients per day

NO, estimate how often

2) In your opinion, how does asylum seeking patients or refugee patients primarily seek help?

$\square$ Of one's own accord (self)

$\square$ Referred from dental care

Referred from health care

$\square$ Other:

3) How aware are you about the effects of torture for the victim?

$\square$ Very aware

$\square$ Aware

Somewhat aware

Not aware at all

4) How common do you think that torture against the oral cavity/face is?

$\begin{array}{ll}\square \text { Very common } & \square \text { Common } \\ \square \text { Not very common } & \square \text { Not common }\end{array}$

5) Is torture something that you have gained knowledge about through your education or at your workplace?

$\square$ Yes, I have received information about torture at my workplace

$\square$ Yes, I have received information about torture through my education

$\square$ We have discussed the subject to some extent (at the workplace or through education)

$\square$ No, I have not received any information about the subject at my workplace

$\square$ No, I have not received any information about the subject through my education

6) Is being a torture victim a reason to seek asylum?

$\square$ Yes $\square$ No

Information about you:

- Age:

- Number of years as a professional:

- Profession:

Working today in:

Public Sector

Private Sector 
cording to Graneheim and Lundman (Graneheim, UH., \& Lundman, B. (2004), and is a suitable method to reveal variation in content (Krippendorff, K., (2004)). Content analysis is a research tool used to determine the presence of certain words, themes, or concepts within some given qualitative data i.e., text from the interviews. The transcribed summary of each interview was processed in four steps (Graneheim, UH., \& Lundman, B. (2004). Step 1: Identify and Collect Data. Step 2: Determine Coding Categories. Step 3: Code the Content. Step 4: Analyse and Present Results. Consensus was reached regarding the informant's knowledge, opinion, or view of the subject. A summary of the selected quotes was sorted in a large table to get a clear picture of all the data, and the interpretation of data was repeatedly discussed. A consensus among the researchers over $80 \%$ should be achieved before categorization is considered complete (Graneheim, UH., \& Lundman, B. (2004). To avoid or at least minimise biased subjectivity in the analysis, the interviews in this study were analysed by not only the interviewers but additional persons.

\section{Results}

Six health care and 16 dental personnel answered the questionnaire, summarized in Table 2a (dental personnel) and Table 2b (health care personnel). One participant from the health care personnel group was unable to find time to meet for an in-person interview and therefore only answered the questionnaire.

\section{Patient contact and multi-professional collaboration}

Health care personnel (5/6) had the impression that patients who have been subjected to torture self-sought care or received a referral via different professionals.
There were referrals from health care centers, psychiatry, employment agencies and SFI (Swedish for immigrants) teachers (Psychologist).

Health care personnel had varying degrees of collaboration with other care units, but rarely with dental care. (2/5) had a collaboration with a dentist and claimed that the rehabilitation time were shortened by this method.

\section{But there should be more collaboration between different caregivers (Dentist)}

None of the health care personnel had been consulted by legitimate dental personnel (dentist, dental hygienist) with questions regarding patients with confirmed or suspected torture injuries. All dental personnel (16/16) experienced a complete lack of collaboration with other disciplines regarding their patients being potential torture victims.

\section{General knowledge and symptoms of torture}

$(4 / 5)$ of the health care personnel claimed that there was a general lack of knowledge among their health care colleagues of torture in the oral cavity or competent care for these survivors. Most of the dental personnel also reported their own lack of knowledge and a belief that their colleagues have no knowledge of symptoms of torture in the oral cavity. The group dental personnel also expressed some extent difficulty formulating the definition of torture. Health care personnel in this study were consistent in identifying that pain or pain sensitivity in general may be a symptom of having been subjected to torture. Health care personnel (4/5) stated PTSD as a general symptom. (3/5) in the group of health care personnel mentioned impaired concentration, cooperation difficulties and learning problems (e.g., inability to learn a new language). 
Table 2. Questions to dental and health care personnel

1. Do you, at a daily basis, meet asylum seekers or patients with a refugee background?

2. What do you find that asylum-seeking patients or patients with a refugee background are mainly seeking help for?

3. How aware are you of what torture means?

4. How common is torture against the oral cavity/face?

5. Are injuries caused by torture something you have come to know about in your education or workplace?

6. Is torture a reason for getting asylum?

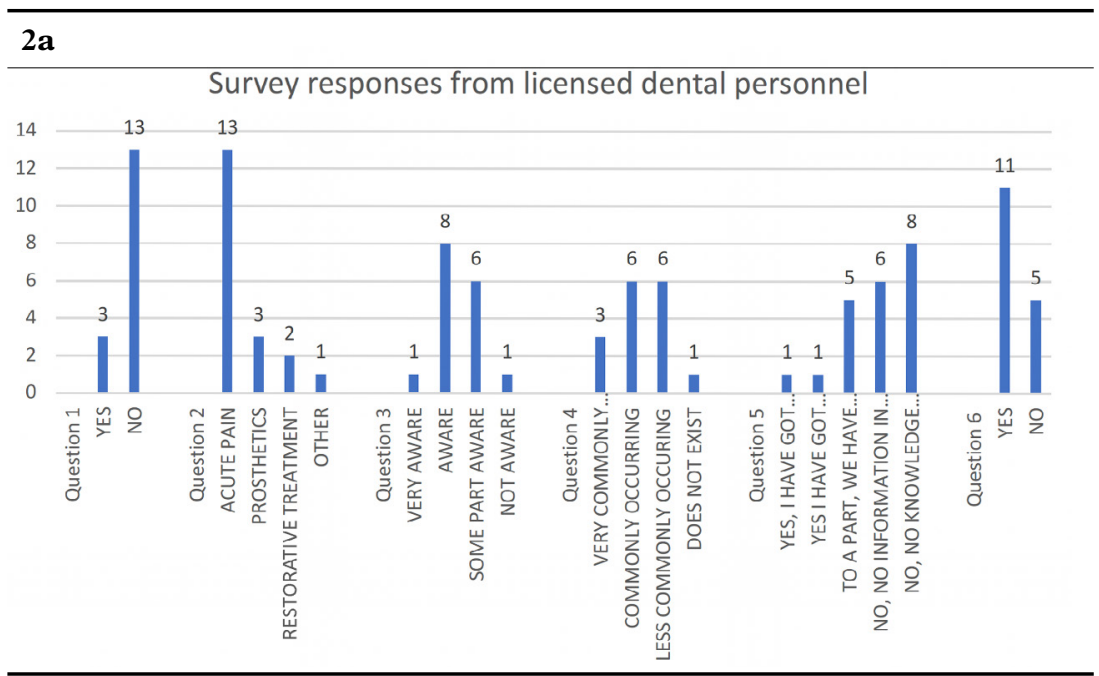

$2 \mathbf{b}$

Survery responses from health care personnel
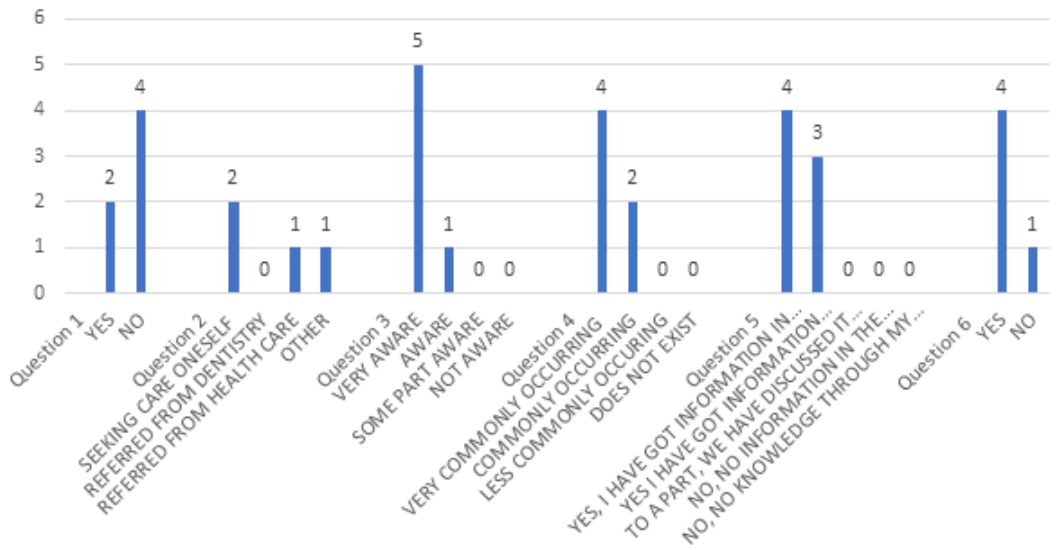
(3/5) health care personnel considered that rapid mood swings, alternating reactions and aggression can be typical symptoms. Other symptoms that the group of health care personnel generally stated were associated with torture included impairment of daily and social functioning, being underweight due to difficulty eating, and having root remnants and missing teeth. All the interviewed dental personnel (16/16) had thoughts on what symptoms patients who have been subjected to torture may exhibit. These included difficultly absorbing information, fear, and the inability to trust others.

How common are injuries in the oral cavity from torture and what are signs of these injuries?

Health care personnel had varying opinions of how common it is for torture survivors to present with injuries in and around the oral cavity due to torture. One participant estimated that about one third of the patients she met had torture injuries in or around the oral cavity. (3/5) cited that the number of survivors with oral cavity injuries due to torture is likely greatly underestimated by providers because health care personnel do not examine the mouth, and therefore probably miss a lot of relevant information. All interviewed dental personnel stated that, as far as they know, they had never met a patient who had been subjected to torture and could not estimate how common it is for their patients to have injuries in and around the oral cavity as a result of torture. One of the interviewed dentists, who works in the public dental sector, stated that he treats all asylum-seeking patients within the area of his clinic but has never seen injuries to the oral cavity caused by torture. (3/5) health care personnel stated that they believed that poor oral hygiene and a high need for dental treatment are general symptoms in torture victims. (3/5) of the health care per- sonnel stated that dental fear is a general symptom and (2/5) mentioned pain, toothache, bruxism/teeth grinding and burn marks as general symptoms experienced by torture survivors. Dental personnel expressed a wide variety of responses concerning damage and possible symptoms to the oral cavity due to torture, including missing teeth, root remnants, necrotic teeth, tooth fractures, poor dental status, wounds, extreme tooth wear and mucosal scarring.

Burn marks in the oral mucosa, teeth extracted with remaining roots in the jaw, classic trauma injuries, extreme tooth attrition due to stress (Dentist)

Knowledge of how to treat torture victims with a focus oral cavity

Health care and dental participants generally considered that their own and their colleagues' knowledge of the importance of the oral cavity in torture is inadequate. The interviewed dental personnel claimed that they can restore good functionality and perform restorative treatment on patients with torture injuries. They indicated that the psychological care of these patients would vary depending on geographically where the patient was able to receive care, including whether the care was in the suburbs or in the inner city. The group of dental personnel also argued that it would be preferred if the patient themselves informed the dental staff about their torture background. Although, as the interviewees stated, this can be hard to achieve since torture is often a very personal and sensitive subject.

Further processing, referring and treatment Health care personnel showed good knowledge of how to refer a torture victim to dental 
personnel, while none of the dental personnel were sure how to refer these patients to other health care and humanitarian professionals. However, dental personnel presented relevant suggestions for possible places to refer their patients, such as the National Board of Health or the Red Cross, and to the general psychiatry, medical specialists in ear-nose and throat, and physiotherapist professionals.

\section{Discussion}

The aim of the study was to investigate dental and health care personnel's awareness of signs displayed in victims of torture with focus on the oral cavity. The main findings were that dental personnel rarely have the education, knowledge, or experience needed to detect signs of torture to the oral cavity. Interviewed dental personnel in this study also expressed a total absence of multidisciplinary collaboration regarding patients who have been tortured. Most of the health care personnel had clear suggestions for external signs of torture and showed good knowledge of how to refer a torture victim to dental personnel.

\section{Recommendation for referral protocol}

Our results show the importance of clear guidelines regarding the management and handling of suspected victims of torture, with a focus on the oral cavity. Centers for tortured refugees should collaborate with dental personnel who have been appropriately trained, can provide any treatment needed, have knowledge of the patient's situation and can provide oral health with a reduced risk of traumatization. Most of the health care personnel in the present study had broad knowledge and competence in identifying signs of torture and showed good knowledge of how to refer a torture victim to dental personnel. Health care teams can identify, train, and cultivate ongoing working relationships with dental personnel in their region to serve survivors and refer torture victims to trained dental personnel, so the patient does not have to seek dental care himself and end up in in the general dentistry. Health care staff can include in their investigation what type of torture has affected the person's mouth, in what way and how much it affects the patient (if it is a trigger for PTSD for example). Information that can be included in a referral to dental personnel is best practice in obtaining patient consent and release of information, and how to prepare their torture survivor clients to receive dental care. Studies show that several aspects of a dental visit can resemble torture methods (Hoyvik et alt (2019), Singh et alt (2008), Keller et alt (2014)). Victims of torture who have been exposed to methods including water, for example waterboarding, may react strongly to water pulsations affecting the oral cavity during treatment. The oral light lamp from the dental chair directed towards a patient's mouth and face may cause the patient to relive memories from interrogations where bright lights were used. As a dental patient you are expected to lie down in the dental chair, which can provoke feelings of loss of control. These situations may trigger the torture victim to relive previous traumatic experiences (Høyvik, A., Lie, B., \& Willumsen, T. (2019).

Trained dental personnel shall give the torture victims as much control as possible over the dental visit and situation, giving them the opportunity to ask questions or, if possible, to alter the environment, e.g., shutting their eyes, or if it can be tolerated and is experienced as relaxing, putting a lavender scented eye pillow over their eyes to counteract the impact of having bright lights shining in their faces.

\section{Improvement actions}

Both dental and health care personnel identified additional problems, such as lack of 
finances and time needed to provide appropriate and sensitive care to torture victims. This corresponds well with the results of previous studies of Singh et alt (2008) and Lamb et alt (2009). The dental personnel interviewed in this study had a broad range of experiences and represented both dentists and dental hygienists with varied work experience, time in the profession, age, background, location of practice (i.e., suburb vs. inner city). None of the dental professionals reported experience with patients who have been - as far as they know - victims of torture. Regarding education, there are very simple means that can be used to improve the knowledge of dental staff, since these issues are not included in undergraduate education today. To begin with, a course should be established within the dental program which covers the complexity of symptoms, etiology, epidemiology, and picture s of injuries, etc. An equivalent course should also be available for medical students and, possibly, cooperation between the two programs could be proposed. As things currently stand, doctors have very limited knowledge about the oral cavity, relying on dental staff. While this is to be expected, health personnel should be equipped to identify when there are issues and make appropriate referrals to dental personnel.

\section{Conclusion}

Survivors of torture carry a risk of reliving the trauma during a visit to the dentist, a situation that may trigger a recollection of torture. Our findings have provided a foundation for understanding the difficulties that torture survivors can experience in receiving dental care. Health care personnel have, in general, good knowledge of how to refer a torture victim to dental personnel, while the dental personnel interviewed in this study expressed uncertainty regarding how to identify a patient as a torture victim and how to refer these patients to other health care and humanitarian professionals. There is a need for increased awareness and knowledge of injuries and signs resulting from torture in the oral cavity among dental and health care personnel. Distinct directives, clear guidelines, education, increased multi-professional collaboration and clear consultation paths are needed.

\section{References}

Abu-Awwad, M., AL-Omoush, S., Shqaidef, A., Hilal, N., \& Hassona, Y. (2020). Oral health-related quality of life among Syrian refugees in Jordan: a cross-sectional study. International Dental fournal, 70, 45-52. doi: 10.1111/idj.12521

Arge, SO., Hansen, SH., \& Lynnerup, N. (2014).

Forensic odontological examinations of alleged torture victims at the University of Copenhagen 1997-2011. Torture, 24, 17-24

Caldas, IM., Magalhaes, T., Afonso, A., \& Matos, E. (2010). The consequences of orofacial trauma resulting from violence: a study in Porto. Dent Traumatol., 26, 484-9. doi: 10.1111/j.16009657.2010.00936.x

Di Napoli, A., Baglio, G., Bracci, C., Taviani, A., Zerbino, E., \& Romano, V. (2005). Torture survivor asylum seekers in Italy: the experience of the humanitarian association Doctors Against Torture, 17, 343-50

Graneheim, UH., \& Lundman, B. (2004). Qualitative content analysis in nursing research: concepts, procedures and measures to achieve trustworthiness. Nurse Education Today, 24, 105112. doi: $10.1016 /$ j.nedt

Høyvik, A., Lie, B., \& Willumsen, T. (2019). Dental anxiety in relation to torture experiences and symptoms of posttraumatic stress disorder. Eur $\mathcal{F}$ Oral Sci., 127, 65-71. doi: 10.1111/eos.12592

Keller, AS., Weiss, J., Resnick, S., Berkowitz, L., Soeprono, A., Sullivan, MJ., Granski, M., Cere, E., \& Wolff, M. (2014). Lessons in health and human rights: providing dental care to torture survivors. F Am Coll Dent., 81, 36-40

Krippendorff, K. (2004. Content analysis. An introduction to its methodology. 2nd ed. Thousand Oaks, London: Sage Publications Ltd.

Lamb, CEF., Whelan, AK., \& Michaels, C. (2009). Refugees and oral health: lessons learned from stories of Hazara refugees. Australian Health Review, 33, 618-27. doi: 10.1071/ah090618

Ozkalipci, Ö., Sahlin, U., Baykal, T., Fincanci, 
SK., Akhan, O., Öztop, F., \& Lök, V. (2010).

Atlas of torture, use of medical and diagnostic examination results in medical assessment of torture. Human Rights of Turkey Publications, 68. Ankara

Singh, HK., Scott, TE., Henshaw, MM., Cote, SE., Grodin, MA., \& Piwowarczyk, LA. (2008). Oral

Health status of refugee torture survivors seeking care in the United States. Am F Public Health., 98, 2181-2. doi: 10.2105/AJPH.2007.120063

UN Office of the High Commissioner for Human Rights. Manual on the Effective Investigation and Documentation of Torture and Other Cruel, Inhuman or Degrading Treatment or Punishment (Istanbul Protocol). (2004)

World Medical Association. WMA Declaration of Helsinki - Ethical Principles for Medical Research Involving Human Subjects. (2021). https://www.wma.net/policies-post/wmadeclaration-of-helsinki-ethical-principles-formedical-research-involving-human-subjects/ 